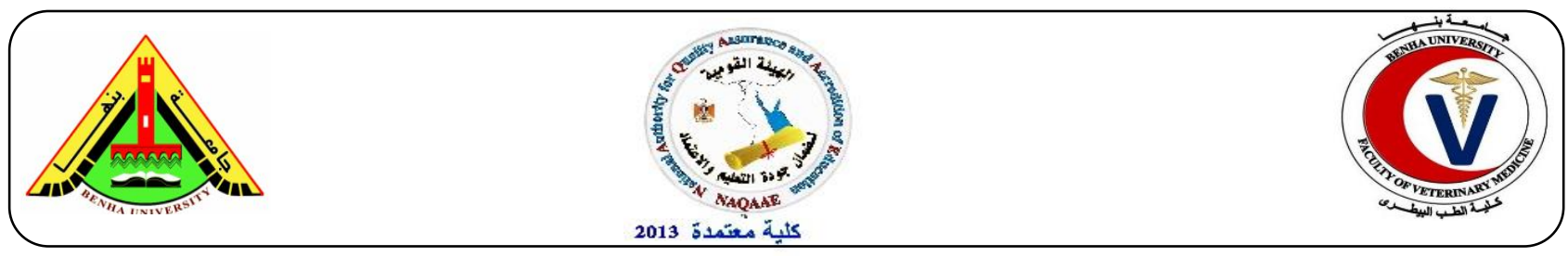

\title{
Evaluation of levamisole efficacy as immune-stimulant on respiratory diseases treatment in calves
}

Fararh K.M.*, Abd EL-Hamied S.S. **, Farid A.S.*and El-Sharkawy R.B.**

Clinical Pathology Dep. Fac. Vet.Med. Benha Univ. *,

\author{
Animal Health Research Institute (Zagazig provincial Lab.) **
}

\section{A B S T R A C T}

This study was performed on fifteen newly born calves, 3-5 month old with clinical signs of bovine respiratory disease (BRD) in addition to five clinically healthy which considered as control group. After bacterial isolation and identification of the causative microorganisms, fifteen calves from which were found infected with E.coli, P. multocida, Klebsiella and S. aureus. The diseased calves were divided randomly into three equal groups: Gp.1: were treated dose of draxxin (2.5 $\mathrm{mg} / \mathrm{kg}$ body weight) by s/c injection in the neck; Gp.2: were received Draxxin and Meloxicam (single $\mathrm{I} / \mathrm{M}$ injection of $0.5 \mathrm{mg} / \mathrm{kgb}$.wt.), while Gp.3: were received draxxin (single s/c injection of $20 \mathrm{mg} / \mathrm{kg}$ bwt.) +Levamisole (single I/M injection at a dose of $1 \mathrm{mg} / 50 \mathrm{~kg}$ bwt). Blood and serum samples were collected from all calves just before treatment, 3 and 10 days post treatment. TLC, neutrophils and monocyte count revealed significant decrease in all treated groups in comparison with diseased group before treatment. While, lymphocyte count recorded significant increase in all treated groups compared with diseased group and lastly, esinophil recorded significant decrease in treated groups with draxxin plus meloxicam and draxxin plus levamisole in comparison with diseased group. Total protein demonstrated significant increase in all treated in comparison with diseased group. Serum albumin, total globulin, $\alpha$-globulin, $\beta$-globulin and $\gamma$-globulin showed significant decrease when compared with diseased calves before treatment. Haptoglobin and fibrinogen showed significant decrease in all treated groups in comparison with diseased group. There were significant increase in immunoglobulins and cellular immunity (phagocytic index and phagocytic percentage) in all treated groups especially in group treated with draxxin plus levamisole when compared with diseased group before treatment.

Keywords: Bovine respiratory disease, Levamisole, Haptoglopin, Fibrinogen, Non-steroidal antiinflammatory drug.

\section{INTRODUCTION}

Diseases of the respiratory system are a major cause of illness and death in cattle from 6 weeks to two years of age. Sadly, this is as true today as it was 30 years ago despite development of new and improved vaccines, new broad spectrum antibiotics, and increased fundamental knowledge as to the cause of disease (Kasimanickam, 2010).
A wide variety of antibacterial agents, most of which are antibiotics are employed for the prevention and treatment of infection in livestock. These antibiotics can cause various problems, such as residues, the emergence of bacterial resistance as well as suppression to the host resistance. A depressed immune function causes marked increase in the incidence of opportunistic 
infection (Nakagawa et al., 1993). Initial field trials with a tulathromycin (Draxxin) suggested a high level of efficacy in treatment of respiratory diseases in newly born calves (Ragbetli et al., 2009).

NSAIDs also have an antiinflammatory effect, but are not immunosuppressive. Some of them have been used with therapeutic success in naturally occurring BRD (Lockwood et al., 1996 and Balmer et al., 1997).

Many drugs have been also used recently to increase the resistance of animals

\section{MATERIALS AND METHODS}

\subsection{Experimental Animals:}

Twenty newly born calves, up to 3 month old belonging to a private farm in Sharkia province, from December till february were used in this study. Five of these animals were apparently healthy and free from any external parasites and kept as a control group. The remaining calves were suffering from bronchopneumonia (fever over $40{ }^{\circ} \mathrm{C}$, cough, nasal discharge from mucous, mucopurulent to purulent, dyspnea and anorexia).The first group were treated with long acting Draxxin; the second group were received Draxxin and Meloxicam (NSAID) while, the third group were received Draxxin and Levamisole.

\subsection{Drug:}

\subsubsection{Draxxin ${ }^{\circledR}$}

Tulathromycin

contain $100 \mathrm{mg} / \mathrm{ml}$ tulathromycin in propylene glycol/water injectable solution ready for use. The recommended dose is a single dose of $2.5 \mathrm{mg} / \mathrm{kg}$ bwt by s/c injection in beef and non-lactating dairy cattle (Skogerboe et al., 2005).

2.2.2. Meloxicam (Metacam) ${ }^{\circledR}$

Solution for injection contains 20 $\mathrm{mg} / \mathrm{ml}$ meloxicam produced by Boehringer Ingelheim Vetmedica Compan, Germany. The recommended therapeutic dose is a single intramuscular injection at a dose of 0.5 $\mathrm{mg} / \mathrm{kg}$ of body weight (Friton et al., 2005).

2.2.3. Levamisole $^{\circledR}$ by improving the humoral and cell mediate immune response such as levamisole hydrochloride (Hany et al., 1999).

The aim of this study was to evaluate bovine respiratory disease (BRD) in calves through using immune-stimulant drug as alternative drug and other drugs on calves suffering from naturally occurring bronchopneumonia and the reflection of these drugs on: leukogram, some inflammatory markers and lastly, humeral and cellular immunity

It's commercial product used as antihelminthic and immune-stimulant for large animals in farms. Levamisole injection(B.P.Vet 85) , Levamisole $\mathrm{HCl} 75$ $\mathrm{mg} / \mathrm{ml}$. The recommended therapeutic dose is A single intramuscular injection at a dose of $1 \mathrm{mg} / 50 \mathrm{~kg}$ b.wt.(Egyptian Co. for Chemical and Pharmaceutical-ADWIA, $10^{\text {th }}$ of Ramadan City (Pekmezci and Cakiroglu , 2009).

2.2.4. Isolation and identification of bacteria:

Nasal swap samples were collected following the antisepsis procedure to prevent possible bacterial contamination risk and placed in transport mediums (Eurotubo collection swab, Delta lab, Spain). Swab sample were cultured in 5\% sheep blood agar and MacConkey Agar. Plates were inoculated and incubated aerobically and anaerobically at $37^{\circ} \mathrm{C}$ for 24-72 hours. Bacteria were identified (Ozkanlar et al., 2012). Streptococcus spp. and Staphylococcus spp. growing in the media were examined in terms of colony structure, Gram staining, microscopic appearance.

\subsubsection{Blood samples :}

Blood samples were collected from the jugular vein of each animal just before treatment, 3 and 10 day post treatment. Sample (1) was $2 \mathrm{ml}$ of blood collected in tube containing heparin $(50 \mathrm{i} . \mathrm{u} / \mathrm{ml})$ used for plasma fibrinogen estimation and cellular immunity tests. Sample (2) was $1 \mathrm{ml}$ of blood collected on di-potassium salt of EDTA for hematological examination. Sample (3) was 3 
$\mathrm{ml}$ of blood taken without anticoagulant collected in a clean and dry tube and left to clot at room temperature for about 2 hours and centrifuged at $3000 \mathrm{rpm}$ for $15 \mathrm{~min}$. Serum samples were drawn in dry clean capped tubes and kept in deep freeze at -20 ${ }^{\circ} \mathrm{C}$ for protein electrophoresis, $\operatorname{IgG}, \operatorname{IgM}$ and estimation of serum haptoglobin.

\section{I- Hematological studies:}

Total and differential leukocytic counts determined by automatic vet CBC counter (Sysmex xt 2000 iv Corporation, KOBE, Japan) in Animal Health Research Institute (Zagazig provincial Lab.) according to the manufacturer instructions.

\section{II-Acute phase proteins:}

-Plasma fibrinogen concentrations was measured according to a method of (Becker et al., 1984).

- Serum haptoglobin was determined photometrically as described by Makimura and Suzuki (1982).

III-Immunological studies :

- Humoral immune response

a- Immunoglobulin:

Serum samples were used for determination of immunoglobulin $\mathrm{M}$ ( $\operatorname{IgM}$ ) according to Naito (1986), while immunoglobulinG (IgG) were measured according to Dati et al. (1989).

b- Proteinogram:

Total protein was measured in Animal Health Research Institute (Dokki, Lab.) according to Henry (1964). Electrophoretic analysis was carried out for determination of serum albumin, alpha, beta and gamma globulins according to the technique described by Davis (1964).

- Cellular immune response:

Phagocytic activity and phagocytic index:

a) Separation of peripheral blood mononuclear cells:

Peripheral blood mononuclear cells (PBMC) were isolated according to the method described by Goddeeris et al. (1986). In brief, $2 \mathrm{ml}$ heparinized blood was harvested from each calf and then diluting the blood 1:2 in heparinized (10 I.U. heparin/ml) PBS. The diluted blood was overlaid on the surface of lymphocyte separation medium FicollHypaque (1:1) in sterile centrifuge tubes, and centrifuged for $15 \mathrm{~min}$ at $80 \mathrm{xg}$. The PBMC was aspirated by $5 \mathrm{ml}$ pipette from the interphase layer, diluted with an equal volume of heparinized PBS and pelleted by centrifugation at $800 \mathrm{xg}$ for $15 \mathrm{~min}$. The PBMC was washed three times $(3 \mathrm{x})$ in heparinized PBS by repeated pipetting with 10 $\mathrm{ml}$ heparinized PBS, resuspension and centrifugation.

b) Phagocytic Assay:

To assess the cell phagocytic activity, was added $0.25 \mathrm{ml}$ of adjusted viable leukocytes suspension to $0.25 \mathrm{ml}$ heat inactivated $C$. albicans in a sterile plastic tubes. The tubes were incubated at $37^{\circ} \mathrm{C}$ for 30 minutes in a humidified $\mathrm{CO}_{2}$ incubator. Subsequently, the tubes were centrifuged at $2500 \mathrm{rpm}$ for 5 minutes and the supernatant was removed with Pasteur pipette leaving a drop .Smears were prepared from the deposit, dried in air and stained with Leishman`s stain.

c) Evaluation of phagocytic activity:

Under a light microscope using oil immersion lens, a total number of 100 phagocytic cells were counted randomly in about ten microscopic fields. The number of ingested yeast cells in each individual phagocytes were determined to calculate the phagocytic cell activity in each of the tested group. The phagocytic-activity is considered as the percentage of phagocytic cells by microscope field. The phagocytic-index is the mean number of C.albicans, ingested by one phagocytic cell.

Statistical analysis:

Statistical analysis was performed using the statistical software package SPSS for windows (Version 18.0; SPSS Inc., Chicago, IL). The significance of differences between the experimental groups were evaluated by one-way analysis of variance (ANOVA) (Kinnear and Gray, 2006). 


\section{RESULTS}

3.1. Results of bacteriological examination:

The bacteriological examination of the cultured swabs from the diseased animals revealed that the isolated bacterial pathogens were E.coli, Pasteurella spp., Klebsiella and Staph Aureus .Table (1).

3.2.Leukogram results:

Concerning to leukogram, diseased calves with BRD showed leukocytosis associated with neutrophilia, monocytosis, eosinophilia and lymphocytopenia before treatment. Treated calves with draxxin in regard to TLC, eosinophil and monocyte and neutrophils count revealed significant decrease, lymphocyte count and basophil recorded non-significant changes when compared with diseased group. While, treated groups with draxxin plus meloxicam and draxxin plus levamisole showed significant decrease in TLC, eosinophil, monocyte and neutrophils count decreased, lymphocyte count showed significant increase when compared with infected control group .Table (2\&3).

\subsection{Protein electrophoresis results:}

The protein electrophoresis of diseased calves showed significant decrease in total protein and albumin. Total protein demonstrated significant increase in all treated groups especially in group treated with draxxin plus levamisole when compared with diseased group. Serum albumin showed significant increase while, total globulin, $\alpha$-globulin, $\beta$ globulin and $\gamma$-globulin showed significant decrease in group treated with draxxin plus meloxicam when compared with diseased calves. Table (4\&5).

3.4.Inflammatory markers results:

Diseased calves suffering from pneumonia revealed significant increase in haptoglobin and fibrinogen. Haptoglobin and fibrinogen exhibited significant decrease in all treated groups when compared with infected control group. Table (6\&7).

\subsection{Immunity results:}

Concerning to immunoglobulin ( $\mathrm{IgG}$ and $\operatorname{IgM}$ ) results in diseased calves revealed significant increase when compared with control group. While, in all treated groups showed significant decrease when compared with diseased group before treatment . Cellular immunity (phagocytic index and phagocytic\%) showed significant decrease in diseased groups in comparison with control group . While, in all treated groups revealed significant increase which more pronounced in group treated with draxxin plus levamisole in comparison with diseased group before treatment. Table (8\&9).

Table (1): Prevalence of the isolated microorganisms from the nasopharyngeal swabs of the pneumonic cattle-calves: (Total number of the examined animals $=20$ )

\begin{tabular}{cccccc}
\hline $\begin{array}{c}\text { Isolated } \\
\text { bacteria }\end{array}$ & E.coli & $\begin{array}{c}P . \\
\text { multocida }\end{array}$ & $\begin{array}{c}\text { Klebsiell } \\
a\end{array}$ & S. aureus & Total No. \\
\hline $\begin{array}{c}\text { No. of positive } \\
\text { animals }\end{array}$ & 5 & 8 & 4 & 3 & 20 \\
\hline$\%$ & 25 & 40 & 20 & 15 & 100 \\
\hline
\end{tabular}


Table (2):Leukogram of healthy and diseased calves before and 3 days post treatment $(n=5)$

\begin{tabular}{|c|c|c|c|c|c|}
\hline \multirow{2}{*}{$\begin{array}{c}\text { Groups } \\
\text { Parameters }\end{array}$} & \multirow{2}{*}{$\begin{array}{c}\text { Healthy } \\
\text { control calves }\end{array}$} & \multirow{2}{*}{$\begin{array}{c}\text { Diseased } \\
\text { calves before } \\
\text { treatment }\end{array}$} & \multicolumn{3}{|c|}{ Diseased calves after treatment with } \\
\hline & & & Draxxin & $\begin{array}{c}\text { Draxxin+ } \\
\text { Meloxicam }\end{array}$ & $\begin{array}{r}\text { Draxxin + } \\
\text { Levamisole }\end{array}$ \\
\hline T.L.C. & 9.14 & 13.43 & 11.07 & 10.47 & 11.44 \\
\hline $\mathrm{x} 10^{3} / \mu 1$ & $\pm 0.50^{c}$ & $\pm 0.14^{\mathrm{a}}$ & $\pm 0.35^{b}$ & $\pm 0.20^{\mathrm{b}}$ & $\pm 0.46^{\mathrm{b}}$ \\
\hline Neutrophils & 4.50 & 7.72 & 5.22 & 4.55 & 5.02 \\
\hline $\mathrm{x} 10^{3} / \mu 1$ & $\pm 0.53^{b}$ & $\pm 0.24^{\mathrm{a}}$ & $\pm 0.27^{\mathrm{b}}$ & $\pm 0.21^{\mathrm{b}}$ & $\pm 0.20^{\mathrm{b}}$ \\
\hline Lymphocytes & 3.89 & 3.77 & 4.03 & 4.47 & 5.03 \\
\hline $\mathrm{x} 10^{3} / \mu 1$ & $\pm 0.18^{\mathrm{bc}}$ & $\pm 0.22^{\mathrm{c}}$ & $\pm 0.28^{\mathrm{bc}}$ & $\pm 0.14^{\mathrm{b}}$ & $\pm 0.12^{\mathrm{a}}$ \\
\hline Monocytes & 0.35 & 0.99 & 0.92 & 0.74 & 0.75 \\
\hline $\mathrm{x} 10^{3} / \mu 1$ & $\pm 0.04^{\mathrm{c}}$ & $\pm 0.02^{\mathrm{a}}$ & $\pm 0.03^{\mathrm{a}}$ & $\pm 0.03^{b}$ & $\pm 0.04^{\mathrm{b}}$ \\
\hline Eosinophils & 0.26 & 0.84 & 0.82 & 0.61 & 0.52 \\
\hline $\mathrm{x} 10^{3} / \mu 1$ & $\pm 0.03^{\mathrm{c}}$ & $\pm 0.03^{a}$ & $\pm 0.05^{\mathrm{a}}$ & $\pm 0.03^{b}$ & $\pm 0.05^{b}$ \\
\hline Basophils & 0.14 & 0.11 & 0.09 & 0.10 & 0.12 \\
\hline $\mathrm{x} 10^{3} / \mu 1$ & $\pm 0.02^{\mathrm{a}}$ & $\pm 0.03^{\mathrm{a}}$ & $\pm 0.02^{\mathrm{a}}$ & $\pm 0.03^{\mathrm{a}}$ & $\pm 0.02^{\mathrm{a}}$ \\
\hline
\end{tabular}

Results are expressed as mean \pm S.E.M.

Different superscripts $(\mathrm{a}, \mathrm{b}, \mathrm{c})$ within the same rows indicate significant differences at $P \leq 0.05$.

Table (3):Leukogram of healthy and diseased calves before and 10 days post treatment $(n=5)$

\begin{tabular}{|c|c|c|c|c|c|}
\hline \multirow{2}{*}{$\begin{array}{c}\text { Groups } \\
\text { Parameters }\end{array}$} & \multirow{2}{*}{$\begin{array}{l}\text { Healthy } \\
\text { control calves }\end{array}$} & \multirow{2}{*}{$\begin{array}{l}\text { Diseased } \\
\text { calves before } \\
\text { treatment }\end{array}$} & \multicolumn{3}{|c|}{ Diseased calves after treatment with } \\
\hline & & & Draxxin & $\begin{array}{l}\text { Draxxin+ } \\
\text { Meloxicam }\end{array}$ & $\begin{array}{l}\text { Draxxin + } \\
\text { Levamisole }\end{array}$ \\
\hline T.L.C. & 9.04 & 13.43 & 10.13 & 9.78 & 9.85 \\
\hline $\mathrm{x} 10^{3} / \mu 1$ & $\pm 0.18^{\mathrm{c}}$ & $\pm 0.14^{\mathrm{a}}$ & $\pm 0.26^{\mathrm{b}}$ & $\pm 0.27^{\mathrm{b}}$ & $\pm 0.22^{b}$ \\
\hline Neutrophils & 3.83 & 7.72 & 4.06 & 3.95 & 4.01 \\
\hline $\mathrm{x} 10^{3} / \mu 1$ & $\pm 0.11^{\mathrm{b}}$ & $\pm 0.24^{\mathrm{a}}$ & $\pm 0.09^{\mathrm{b}}$ & $\pm 0.24^{\mathrm{b}}$ & $\pm 0.19^{b}$ \\
\hline Lymphocytes & 4.37 & 3.77 & 4.29 & 4.63 & 4.49 \\
\hline $\mathrm{x} 10^{3} / \mu 1$ & $\pm 0.29^{\mathrm{a}}$ & $\pm 0.22^{b}$ & $\pm 0.11^{\mathrm{ab}}$ & $\pm 0.06^{\mathrm{a}}$ & $\pm 0.28^{\mathrm{a}}$ \\
\hline Monocytes & 0.47 & 0.99 & 0.90 & 0.53 & 0.77 \\
\hline $\mathrm{x} 10^{3} / \mu 1$ & $\pm 0.04^{\mathrm{d}}$ & $\pm 0.02^{\mathrm{a}}$ & $\pm 0.04^{\mathrm{b}}$ & $\pm 0.05^{\mathrm{d}}$ & $\pm 0.04^{\mathrm{c}}$ \\
\hline Eosinophils & 0.27 & 0.84 & 0.79 & 0.58 & 0.50 \\
\hline $\mathrm{x} 10^{3} / \mu \mathrm{l}$ & $\pm 0.14^{\mathrm{c}}$ & $\pm 0.03^{a}$ & $\pm 0.04^{\mathrm{a}}$ & $\pm 0.03^{b}$ & $\pm 0.04^{\mathrm{b}}$ \\
\hline Basophils & 0.10 & 0.11 & 0.09 & 0.09 & 0.08 \\
\hline $\mathrm{x} 10^{3} / \mu 1$ & $\pm 0.01^{\mathrm{a}}$ & $\pm 0.03^{\mathrm{a}}$ & $\pm 0.02^{\mathrm{a}}$ & $\pm 0.02^{\mathrm{a}}$ & $\pm 0.02^{\mathrm{a}}$ \\
\hline
\end{tabular}

Results are expressed as mean \pm S.E.M.

Different superscripts $(\mathrm{a}, \mathrm{b}, \mathrm{c})$ within the same rows indicate significant differences at $P \leq 0.05$. 
Table (4):Proteinelectrophoresis of healthy and diseased calves before and 3 days post treatment $(\mathrm{n}=5)$

\begin{tabular}{|c|c|c|c|c|c|}
\hline \multirow{2}{*}{$\begin{array}{l}\text { Groups } \\
\text { Parameters }\end{array}$} & \multirow{2}{*}{$\begin{array}{c}\text { Healthy } \\
\text { control calves }\end{array}$} & \multirow{2}{*}{$\begin{array}{c}\text { Diseased } \\
\text { calves before } \\
\text { treatment }\end{array}$} & \multicolumn{3}{|c|}{ Diseased calves after treatment with } \\
\hline & & & Draxxin & $\begin{array}{c}\text { Draxxin+ } \\
\text { Meloxicam }\end{array}$ & $\begin{array}{c}\text { Draxxin }+ \\
\text { Levamisole }\end{array}$ \\
\hline Total protein & 7.06 & 7.35 & 7.36 & 7.32 & 7.44 \\
\hline $\mathrm{g} / \mathrm{dl}$ & $\pm 0.06^{\mathrm{b}}$ & $\pm 0.06^{\mathrm{a}}$ & $\pm 0.05^{\mathrm{a}}$ & $\pm 0.05^{\mathrm{a}}$ & $\pm 0.03^{\mathrm{a}}$ \\
\hline Albumin & 3.70 & 3.26 & 3.47 & 3.56 & 3.52 \\
\hline $\mathrm{g} / \mathrm{dl}$ & $\pm 0.03^{\mathrm{a}}$ & $\pm 0.01^{\mathrm{d}}$ & $\pm 0.03^{c}$ & $\pm 0.02^{\mathrm{b}}$ & $\pm 0.02^{b c}$ \\
\hline$\alpha$-Globulin & 0.86 & 1.02 & 0.94 & 0.90 & 0.93 \\
\hline $\mathrm{g} / \mathrm{dl}$ & $\pm 0.02^{\mathrm{c}}$ & $\pm 0.02^{\mathrm{a}}$ & $\pm 0.01^{\mathrm{b}}$ & $\pm 0.02^{b c}$ & $\pm 0.02^{b}$ \\
\hline$\beta$-Globulin & 0.88 & 1.02 & 0.98 & 0.92 & 0.99 \\
\hline $\mathrm{g} / \mathrm{dl}$ & $\pm 0.01^{\mathrm{d}}$ & $\pm 0.02^{\mathrm{a}}$ & $\pm 0.01^{\mathrm{b}}$ & $\pm 0.01^{\mathrm{c}}$ & $\pm 0.01^{\mathrm{a} \mathrm{b}}$ \\
\hline$\gamma$-Globulin & 1.62 & 2.04 & 1.97 & 1.93 & 2.00 \\
\hline $\mathrm{g} / \mathrm{dl}$ & $\pm 0.04^{\mathrm{b}}$ & $\pm 0.04^{\mathrm{a}}$ & $\pm 0.04^{\mathrm{a}}$ & $\pm 0.04^{\mathrm{a}}$ & $\pm 0.03^{\mathrm{a}}$ \\
\hline T-globulin & 3.36 & 4.09 & 3.89 & 3.75 & 3.92 \\
\hline $\mathrm{g} / \mathrm{dl}$ & $\pm 0.06^{\mathrm{d}}$ & $\pm 0.05^{\mathrm{a}}$ & $0.04^{b c}$ & $\pm 0.05^{\mathrm{c}}$ & $\pm 0.04^{b}$ \\
\hline
\end{tabular}

Results are expressed as mean \pm S.E.M.

Different superscripts $(\mathrm{a}, \mathrm{b}, \mathrm{c}, \mathrm{d})$ within the same rows indicate significant differences at $P \leq 0.05$.

Table (5):Proteinelectrophoresis of healthy and diseased calves before and 10 days post treatment $(\mathrm{n}=5)$

\begin{tabular}{|c|c|c|c|c|c|}
\hline \multirow{2}{*}{$\begin{array}{c}\text { Groups } \\
\text { Parameters }\end{array}$} & \multirow{2}{*}{$\begin{array}{c}\text { Healthy } \\
\text { control calves }\end{array}$} & \multirow{2}{*}{$\begin{array}{c}\text { Diseased } \\
\text { calves before } \\
\text { treatment }\end{array}$} & \multicolumn{3}{|c|}{ Diseased calves after treatment with } \\
\hline & & & Draxxin & $\begin{array}{l}\text { Draxxin+ } \\
\text { Meloxicam }\end{array}$ & $\begin{array}{c}\text { Draxxin }+ \\
\text { Levamisole }\end{array}$ \\
\hline Total protein & 7.08 & 7.35 & 7.25 & 7.30 & 7.43 \\
\hline $\mathrm{g} / \mathrm{dl}$ & $\pm 0.04^{\mathrm{c}}$ & $\pm 0.06^{\mathrm{ab}}$ & $\pm 0.04^{\mathrm{b}}$ & $\pm 0.04^{\mathrm{b}}$ & $\pm 0.02^{\mathrm{a}}$ \\
\hline Albumin & 3.74 & 3.26 & 3.51 & 3.65 & 3.59 \\
\hline $\mathrm{g} / \mathrm{dl}$ & $\pm 0.03^{\mathrm{a}}$ & $\pm 0.01^{\mathrm{d}}$ & $\pm 0.03^{c}$ & $\pm 0.02^{\mathrm{b}}$ & $\pm 0.01^{\mathrm{b}}$ \\
\hline$\alpha$-Globulin & 0.85 & 1.02 & 0.92 & 0.88 & 0.90 \\
\hline $\mathrm{g} / \mathrm{dl}$ & $\pm 0.01^{\mathrm{d}}$ & $\pm 0.02^{\mathrm{a}}$ & $\pm 0.01^{\mathrm{b}}$ & $\pm 0.01^{\mathrm{cd}}$ & $\pm 0.01^{\mathrm{bc}}$ \\
\hline$\beta$-Globulin & 0.88 & 1.02 & 0.94 & 0.90 & 0.96 \\
\hline $\mathrm{g} / \mathrm{dl}$ & $\pm 0.01^{\mathrm{c}}$ & $\pm 0.02^{\mathrm{a}}$ & $\pm 0.01^{\mathrm{b}}$ & $\pm 0.01^{\mathrm{c}}$ & $\pm 0.004^{\mathrm{b}}$ \\
\hline$\gamma$-Globulin & 1.61 & 2.04 & 1.87 & 1.86 & 1.97 \\
\hline $\mathrm{g} / \mathrm{dl}$ & $\pm 0.04^{\mathrm{c}}$ & $\pm 0.04^{\mathrm{a}}$ & $\pm 0.02^{\mathrm{b}}$ & $\pm 0.03^{b}$ & $\pm 0.02^{\mathrm{a}}$ \\
\hline $\begin{array}{l}\text { T-globulin } \\
\text { g/dl }\end{array}$ & $\begin{array}{c}3.34 \\
\pm 0.05^{d}\end{array}$ & $\begin{array}{c}4.09 \\
\pm 0.05^{\mathrm{a}}\end{array}$ & $\begin{aligned} & 3.73 \\
\pm & 0.02^{\mathrm{bc}}\end{aligned}$ & $\begin{array}{r}3.64 \\
\pm 0.03^{c}\end{array}$ & $\begin{array}{c}3.83 \\
\pm 0.02^{\mathrm{b}}\end{array}$ \\
\hline
\end{tabular}

Results are expressed as mean \pm S.E.M.

Different superscripts $(\mathrm{a}, \mathrm{b}, \mathrm{c}, \mathrm{d})$ within the same rows indicate significant differences at $P \leq 0.05$. 
Table (6): Inflammatory markers of healthy and diseased calves before and 3 days post treatment $(\mathrm{n}=5)$

\begin{tabular}{|c|c|c|c|c|c|}
\hline \multirow{2}{*}{$\begin{array}{l}\text { Groups } \\
\text { Parameters }\end{array}$} & \multirow{2}{*}{$\begin{array}{c}\text { Healthy } \\
\text { control calves }\end{array}$} & \multirow{2}{*}{$\begin{array}{c}\text { Diseased } \\
\text { calves before } \\
\text { treatment }\end{array}$} & \multicolumn{3}{|c|}{ Diseased calves after treatment with } \\
\hline & & & Draxxin & $\begin{array}{c}\text { Draxxin+ } \\
\text { Meloxicam }\end{array}$ & $\begin{array}{c}\text { Draxxin + } \\
\text { Levamisole }\end{array}$ \\
\hline Haptoglobin & 1.30 & 6.10 & 4.40 & 2.11 & 3.61 \\
\hline$m g / d l$ & $\pm 0.04^{\mathrm{e}}$ & $\pm 0.30^{\mathrm{a}}$ & $\pm 0.07^{b}$ & $\pm 0.09^{\mathrm{d}}$ & $\pm 0.07^{\mathrm{c}}$ \\
\hline Fibrinogen & 512.80 & 844.60 & 746.80 & 551.80 & 621.00 \\
\hline$m g / d l$ & $\pm 1.59^{\mathrm{e}}$ & $\pm 3.14^{\mathrm{a}}$ & $\pm 5.29^{b}$ & $\pm 4.97^{\mathrm{d}}$ & $\pm 5.14^{\mathrm{c}}$ \\
\hline
\end{tabular}

Results are expressed as mean \pm S.E.M.

Different superscripts (a,b,c,d,e) within the same rows indicate significant differences at $P \leq 0.05$.

Table (7): Inflammatory markers of healthy and diseased calves before and 10 days post treatment $(\mathrm{n}=5)$

\begin{tabular}{|c|c|c|c|c|c|}
\hline \multirow{2}{*}{$\begin{array}{l}\text { Groups } \\
\text { Parameters }\end{array}$} & \multirow{2}{*}{$\begin{array}{l}\text { Healthy } \\
\text { control } \\
\text { calves }\end{array}$} & \multirow{2}{*}{$\begin{array}{c}\text { Diseased } \\
\text { calves before } \\
\text { treatment }\end{array}$} & \multicolumn{3}{|c|}{ Diseased calves after treatment with } \\
\hline & & & Draxxin & $\begin{array}{c}\text { Draxxin+ } \\
\text { Meloxicam }\end{array}$ & $\begin{array}{c}\text { Draxxin + } \\
\text { Levamisole }\end{array}$ \\
\hline Haptoglobin & 1.30 & 6.1 & 3.5 & 1.6 & 3.10 \\
\hline$m g / d l$ & $\pm 0.04^{\mathrm{e}}$ & $\pm 0.3^{\mathrm{a}}$ & $\pm 0.09^{\mathrm{b}}$ & $\pm 0.03^{d}$ & $\pm 0.03^{c}$ \\
\hline Fibrinogen & 511.80 & 844.60 & 532.80 & 518.80 & 524.80 \\
\hline$m g / d l$ & $\pm 2.89^{b}$ & $\pm 3.14^{\mathrm{a}}$ & $\pm 8.90^{\mathrm{b}}$ & $\pm 3.62^{\mathrm{b}}$ & $\pm 2.46^{\mathrm{b}}$ \\
\hline
\end{tabular}

Results are expressed as mean \pm S.E.M.

Different superscripts (a,b,c,d,e) within the same rows indicate significant differences at $P \leq 0.05$.

Table (8):Hummoral and cellular immunity of healthy and diseased calves before and 3 days post treatment $(\mathrm{n}=5)$

\begin{tabular}{|c|c|c|c|c|c|}
\hline \multirow{2}{*}{$\begin{array}{l}\text { Groups } \\
\text { Parameters }\end{array}$} & \multirow{2}{*}{$\begin{array}{c}\text { Healthy } \\
\text { control calves }\end{array}$} & \multirow{2}{*}{$\begin{array}{c}\text { Diseased } \\
\text { calves before } \\
\text { treatment }\end{array}$} & \multicolumn{3}{|c|}{ Diseased calves after treatment with } \\
\hline & & & Draxxin & $\begin{array}{c}\text { Draxxin+ } \\
\text { Meloxicam }\end{array}$ & $\begin{array}{c}\text { Draxxin + } \\
\text { Levamisole }\end{array}$ \\
\hline $\operatorname{IgM}$ & 6.14 & 18.71 & 15.35 & 11.59 & 13.68 \\
\hline$g / L$ & $\pm 0.62^{\mathrm{e}}$ & $\pm 0.26^{\mathrm{a}}$ & $\pm 0.47^{\mathrm{b}}$ & $\pm 0.53^{\mathrm{d}}$ & $\pm 0.22^{\mathrm{c}}$ \\
\hline IgG & 2.22 & 7.88 & 7.21 & 4.26 & 4.98 \\
\hline$g / L$ & $\pm 0.24^{c}$ & $\pm 0.37^{\text {a }}$ & $\pm 0.24^{\mathrm{a}}$ & $\pm 0.09^{b}$ & $\pm 0.28^{b}$ \\
\hline Phagocytic & 6.70 & 3.19 & 3.74 & 4.74 & 4.78 \\
\hline index & $\pm 0.12^{\mathrm{a}}$ & $\pm 0.11^{\mathrm{d}}$ & $\pm 0.05^{\mathrm{c}}$ & $\pm 0.09^{b}$ & $\pm 0.08^{b}$ \\
\hline Phagocytic $\%$ & 76.60 & 59.00 & 64.00 & 71.00 & 74.60 \\
\hline Phagocytic \% & $\pm 0.93^{\mathrm{a}}$ & $\pm 0.71^{\mathrm{d}}$ & $\pm 0.55^{c}$ & $\pm 0.95^{b}$ & $\pm 0.51^{\mathrm{a}}$ \\
\hline
\end{tabular}


Results are expressed as mean \pm S.E.M.

Different superscripts (a,b,c,d,e) within the same rows indicate significant differences at $P \leq 0.05$.

Table (9):Hummoral and cellular immunity of healthy and diseased calves before and 10 dayspost treatment $(\mathrm{n}=5)$

\begin{tabular}{|c|c|c|c|c|c|}
\hline \multirow{2}{*}{$\begin{array}{l}\text { Groups } \\
\text { Parameters }\end{array}$} & \multirow{2}{*}{$\begin{array}{l}\text { Healthy } \\
\text { control } \\
\text { calves }\end{array}$} & \multirow{2}{*}{$\begin{array}{c}\text { Diseased } \\
\text { calves before } \\
\text { treatment }\end{array}$} & \multicolumn{3}{|c|}{ Diseased calves after treatment with } \\
\hline & & & Draxxin & $\begin{array}{c}\text { Draxxin+ } \\
\text { Meloxicam }\end{array}$ & $\begin{array}{c}\text { Draxxin } \\
+ \text { Levamisole }\end{array}$ \\
\hline $\operatorname{IgM}$ & 6.38 & 18.71 & 11.19 & 8.33 & 9.26 \\
\hline$g / L$ & $\pm 0.69^{\mathrm{e}}$ & $\pm 0.26^{\mathrm{a}}$ & $\pm 0.42^{b}$ & $\pm 0.27^{\mathrm{d}}$ & $\pm 0.36^{c}$ \\
\hline IgG & 2.10 & 7.88 & 5.54 & 3.45 & 3.56 \\
\hline$g / L$ & $\pm 0.18^{\mathrm{d}}$ & $\pm 0.37^{\mathrm{a}}$ & $\pm 0.16^{b}$ & $\pm 0.19^{c}$ & $\pm 0.20^{\mathrm{c}}$ \\
\hline Phagocytic & 5.76 & 3.19 & 4.16 & 5.00 & 5.16 \\
\hline index & $\pm 0.098^{\mathrm{a}}$ & $\pm 0.11^{\mathrm{d}}$ & $\pm 0.05^{c}$ & $\pm 0.07^{b}$ & $\pm 0.07^{b}$ \\
\hline Phoogenti & 74.00 & 59.00 & 68.80 & 72.43 & 73.68 \\
\hline Pnagocyuc \% & $\pm 0.55^{\mathrm{a}}$ & $\pm 0.71^{\mathrm{c}}$ & $\pm 0.66^{\mathrm{b}}$ & $\pm 0.75^{\mathrm{a}}$ & $\pm 0.43^{\mathrm{a}}$ \\
\hline
\end{tabular}

Results are expressed as mean \pm S.E.M.

Different superscripts (a,b,c,d,e) within the same rows indicate significant differences at $P \leq 0.05$.

\section{DISCUSSION}

The bacteriological examination of the cultured swabs from the diseased animals revealed that the isolated bacterial pathogens were E.coli, Pasteurella spp., Klebsiella and Staph. This results the same as reported by Aytekin et al., (2010).

Concerning to the leukogram, diseased calves with BRD showed leukocytosis associated with neutrophilia, monocytosis, eosinophilia and lymphocytopenia before treatment. Such findings indicated the presence of inflammation and suppuration caused by the bacterial infection and the response of leukogram to the inflammatory lung disease (Coles, 1986). Our findings are similar to those previously cited by Faris et al. (2010) and Eleiwa et al.(2014).

Treated calves with draxxin in regard to TLC, eosinophil and monocyte and neutrophils count revealed significant decrease and lastly, lymphocyte count and basophil recorded nonsignificant changes when compared with diseased group. Similar results obtained by Crew et al. (2013) and Eleiwa et al. (2014). Neutrophilia may occur due to neutrophils provide the first line of defence against pathogens when calves suffer from BRD (Janeway et al., 2005).

Regarding to leukogram in treated groups with draxxin plus meloxicam showed significant decrease in TLC, eosinophil, monocyte and neutrophils count comparativly with diseased group while, lymphocyte count and basophil recorded non-significant changes when compared with diseased group. This results agree with Faris et al.(2010) who attributed this to meloxicam which has a nonsteroidal structure, and acts on the archidonic acid cascade in the inflammatory process by inhibiting the production of prostaglandin $\mathrm{H} 2$ through the enzyme cyclo-oxygenase $(\mathrm{COX})$.While, treated group with draxxin plus levamisole revealed that TLC, neutrophil, lymphocyte, monocyte count and eosinophil recorded significant decrease while, basophil showed non-significant change compared with infected control group. This attributed to that levamisole have cholinergic effect on leukocytes and also increases protein and nucleic acid synthesis in resting leukocytes, 
thus causing leukocytosis Bourne et al. (1978).

The protein electrophoresis of diseased calves showed significant decrease in total protein and albumin. Similar results obtained by Csilla et al. (2013). The significant decrease of serum albumin in diseased calves could be attributed to the destructive effect of bacteria and its toxins on the liver cells which are the main sources of albumin and protein synthesis in the body McPherson (1984). A significant increase in $\alpha$-globulin indicating tissue damage results from infection or inflammation (Faris et al., 2010) .The $\beta$ globulin and $\gamma$-globulin showed higher values indicating the activation of the immune defense of calves due to the infection (Coles, 1986).

Treated groups with draxxin revealed that serum total protein and albumin demonstrated significant increase compared to diseased calves before treatment. Meanwhile, total globulin ( $\alpha$-globulin, $\beta$-globulin and $\gamma$ globulin) revealed significant decrease compared to diseased calves before treatment. Similar results reported by Bednarek et al.(1999).Although many antimicrobial agents have been reported to cause immunesuppression effect in animals, the macrolide antibiotics enhance the immune function by activation of the peripheral blood leukocytes function, cytokine production, increase random migration of neutrophils and their phagocytic activity after one week posttreatment of the bronchopneumonia in calves (Bednarek et al., 1998).

Treated groups with draxxin plus meloxicam the serum albumin showed significant increase and reached to the highest value. Meanwhile, total globulin ( $\alpha$-globulin, $\beta$-globulin and $\gamma$-globulin) revealed significant decrease compared to diseased calves and reached to the lowest value.This results attributed to that meloxicam produce analgesia and reduce inflammation by inhibiting the enzyme of cyclooxygenase (COX) and subsequent prostaglandin production in the peripheral tissues and central nervous system (Friton et al., 2005 and Francoz et al., 2012).
Treated group with draxxin plus levamisole revealed that total protein demonstrated significant increase and recorded the highest value. Serum albumin showed significant increase. Meanwhile, total globulin, $\alpha$-globulin, $\beta$-globulin and $\gamma$-globulin showed significant decrease compared to diseased group. Our finding agree with Garszon (1992) and Holcombe et al.(1998) This results attributed to that levamisole enhance the immune response and act as immunestimulants, immune-potentiators (Blecha, 1988).

Diseased calves suffering from pneumonia revealed significant increase in haptoglobin and fibrinogen. Our results were paralleled to the finding of Angen et al. (2009) who reported that the haptoglobin might be the best choice for detecting respiratory diseases under field conditions and to distinguish between calves requiring antiinflammatory drug.

Treated groups with draxxin alone, draxxin plus meloxicam, draxxin plus levamisole exhibited significant decrease in haptoglobin and fibrinogen in all treated groups which were more pronounced decrease in group treated with draxxin plus meloxicam. Similar results agree with Lynch et al., (2010) and Richeson et al. (2013).

Treated groups with draxxin, draxxin plus meloxicam and lastly draxxin plus levamisole $\operatorname{IgM}$ and $\operatorname{IgG}$ results revealed significant decrease compared to diseased group but reached the lowest value in group treated with draxxin plus meloxicam. These results agree with Perino (1997) and Galyean et al. (1999) who reported that feedlot respiratory morbidity was associated with lower serum plasma protein, but not serum $\mathrm{IgG}$, at $24 \mathrm{~h}$ after birth. Calves with inadequate plasma proteins at $24 \mathrm{~h}$ after birth had a greater risk of morbidity and respiratory tract morbidity in the feedlot compared with calves that had adequate plasma protein.

Concerning to cellular immunity of the diseased calves phagocytic index and phagocytic percent showed significant decrease .This results agree with Chirase et al. (2004) and Carroll et al. (2007). Treated 
group with draxxin plus levamisole results of cellular immunity (phagocytic index and phagocytic\%) revealed significant increase. Similar results were obtained by Bellavite et al. (2006) who reported that levamisole has a broad range of effects on the immune system by stimulating cell mediated immunity through potentiating the rate of T-lymphocyte differentiation, the responsiveness to antigen and mitogens and the activity of effector lymphocytes. Furthermore, levamisole stimulate phagocytosis and chemotaxis for neutophil and monocytes.

\section{CONCLUSION}

It could be concluded that, the use of draxxin plus levamisole as alternative drug in calves which suffered from BRD provide enhancement of cellular immunity. Also, combination of meloxicam with draxxin was superior to the antibiotic alone.

\section{REFERENCES}

Angen O., Thomsen J., Larsen L.E., Kokotovic B., Heegaard P.M. and Enemark J.M. (2009): Respiratory disease in calves: Microbiological investigations on transtracheally aspirated bronchoalveolar and acute phase protein response. Vet. Microbiol., 28: 137(1-2):165-171.

Aytekin I., Mamak N., Onmaz A.C., Sakin F.and Aslan S. (2010): Effects of Tulathromycin and Tilmicosin application in the treatment of bovine respiratory disease in cattle. Veteriner Fakultesi Dergisi., 21(3): 159-162.

Balmer T.V.,Williams P. and Selman I.E. (1997): Comparison of carprofen and flunixin meglumine as adjunctive therapy in bovine respiratory disease. Vet. J., 154: 233-241.

Becker U., Bartl K. and Wahlefed A.W. (1984): A functional photometric assay for plasma fibrinogen. Thromb. Res., 35: 475-484.

Bednarek D., Szuster-Ciesielska A., Zdzisinska B., Kondracki M., Paduch R. and Kandefer-Szersze N.M. (1999): The effect of steroidal and non-steroidal antiinflammatory drugs on the cellular immunity of calves with experimentally induced local lung inflammation. Vet. Immunol. Immunopathol., 71: 1-15.

Bednarek D., Zdzisinska B., Kondracki M., Rzeski W. and Lokaj I. (1998): Alterations in peripheral blood leukocytes functions during enzootic bronchopneumonia of calves.Effect of treatment with antibiotics and immunomodulators. Dtsch Tietarztl Wochenschr., 105 (5):194-199.

Bellavite P., Ortolani R. and Conforti A. (2006): Immunology and homeopathy .3.Experimental studies on animal models .Advance Acess Publication, e CAM, 3 (2):171-186.

Blecha F. (1988): Immunomodulation: A means of disease prevention in stressed livestock. Journal of Animal Science, 66: 2084-2090.

Bourne F.J., Newby T.J., Evans P. and Morgan K. (1978): The immune requirements of the newborn pig and calf. Ann. Vet. Res., 9:239-244.

Carroll J.A. and Forsberg N.E. (2007): Influence of stress and nutrition on cattle immunity. Vet. Clin. North. Am. Food Anim. Pract., 23: $105-150$.

Chirase N.K., Greene L.W., Purdy Ch.W., Loan R.W., Auvermann B.W., Parker D.B., Walborg E.F., Stevenson D.E., Xu Y. and Klauning J.E. (2004): Effect of transport stress on respiratory disease, serum antioxidant status and serum concentrations of lipid peroxidation biomarkers in beef cattle. American Journal of Veterinary Research, 65: 860864.

Coles E.H. (1986): Veterinary Clinical Pathology. $4^{\text {th }}$ Ed.W.B. Saundres Company,Philadelphia London and Toronto.

Crews C.E., Powell J.G., Kegley E.B., Reynolds J.L., and Hornsby J.A. (2013): Isoflupredone acetate as ancillary therapy for bovine respiratory disease in high-risk stocke calves. University of Arkansas System Division of Agriculture, Department of Animal Science, Fayetteville, Ark. 
Csilla T., Oskar N. and Gabriel K. (2013): The serum protein electrophoretic pattern and acute phase proteins concentrations in calves with chronic respiratory diseases. Acta Veterinaria (Beograd), 63 (5-6): 473-486.

Dati F. (1989): Lab. Med. Clin. Immunoassay, 13,87 .

Davis B.J. (1964): Disk electrophoresis, Methods and application to human serum proteins. J. Am. New York Acad. Sci., 121: $404-428$.

Eleiwa N.Z.H., Gad G.N.A. and EL-Shorbagy A.I.A.(2014): Effect of a combined Oxytetracycline $\mathrm{HCl} /$ Flunixin meglumine therapy on undifferentiated respiratory disease in calves. International Journal of Advanced Research, 2 (3): 387-398.

Faris A.E.S., Abd EL-Hamied S.S., Mohamed M.M. and Refaat S.A. (2010): A comparison on the effect of steroidal and non-steroidal anti-inflammatory drugs with antibiotic treatment of respiratory diseases in calves.Suez Canal Veterinary Journal, 1110-6298.

Francoz D., Buczinski S. and Apley M. (2012): Evidence related to the use of ancillary drugs in bovine respiratory disease (anti-inflammatory and others): are they justified or not? Vet Clin Food Anim., 28:23-38.

Friton G.M., Cajal C.and Romero R.R. (2005): Long-term effects of meloxicam in the treatment of respiratory disease in fattening cattle. Vet. Rec. 156:809-811.

Galyean M.L., Perino L.J. and Duff G.C. (1999): Interaction of cattle health/immunity and nutrition. J. Anim. Sci., 77:1120-1134.

Garszon M.C. (1992): Levamisole treatment in HIV - infected Zambian children. Lancet, 340: 1099-1100.

Goddeeris B.M., Baldwin C.L., Ole-MoiYoi O. and Morrison W.I. (1986): Improved methods for purification and depletion of monocytes from bovine peripheral blood mononuclear cells: Functional evaluation of monocytes in response to lectins. J. Immunol. Method, 89: 165170.

Hany A.A., Susan T.K., Nabil A.A. and Madkour M.S. (1999): Effect of levamisole as an immunostimulant on infectious bursal disease virus vaccination. J. Vet. Med. Res., 1:45-57.

Henry R.J. (1964): Colorimetric determination of total protein. Clinical chemistry, Harper \& Row Publishers,New York pp. 181.

Holcombe R.F., Li A., Stewart R.M. (1998): Levamisole and interleukin-2 for advanced malignancy. Biotherapy, 11 (14): 255-258.

Janeway C.A., Travers M., Walport M. and Shlomchik M.J. (2005): Immunobiology, the immune system in health and disease. Garland Science Publications, New York, 6.

Kasimanickam R. (2010): Bovine respiratory disease "shipping fever" in cattle. Washington State University .Veterinary Medicine Extension .p. 1-3.

Kinnear P. and Gray C. (2006): SPSS 12 made simple. Psychololgy press.

Lockwood P.W., Johnson J.C. and Johnson E.D. (1996): Flunixin meglumine as an adjunct to antibacterial therapy: efficacy in the treatment of the bovine respiratory disease complex under typical North American feedlot conditions. In: Proceedings of the XIX World Buiatric Congress, Edinburgh, pp. 50-53.

Lynch E.M., Earley B., McGee M. and Doyle S. (2010): Effect of abrupt weaning at housing on leukocyte distribution, functional activity of neutrophils, and acute phase protein response of beef calves. BMC Veterinary Research, pneumonia.Journal and veterinary Advances, 8 (11): 2345-2349.

Makimura S. and Suzuki N. (1982): Quantitative determination of bovine serum haptoglobin and its elevation in some inflammatory diseases. Jpn. J. Vet. Sci., 44:15-21.

McPherson R.A. (1984): Specific protein in: Clinical Diagnosis and Management by 
Evaluation of levamisole efficacy as immune-stimulant on respiratory diseases treatment in calves

Laboratory Methods. Edited by Hendry, J.B.; Saunders, W.B. Philadelphia.

Naito H.K.(1986): J.Clin.Immunoassay ,9,155.

Nakagawa J., Osame S., Ichijo S., Araki S. and Kimura M. (1993): Effect of active egg white product on neutrophil function in calves. J. Vet. Med. Sci., 5: 259-263.

Ozkanlar Y., Aktas M.S., Kaynar O., Ozkanlar S., Kirecci E. and Yildiz L. (2012): Bovine respiratory disease in naturally infected calves:clinical signs,blood gases and cytokine response. Revue Méd. Vét., 163 (3): 123-130.

Pekmezci D. and Cakiroglu D. (2009): Investigation of Immune modulatory effects of levamisole and vitamin $\mathrm{E}$ on Immunity and some blood parameters in newborn Jersey calves. Vet Res Commun., 33:711-721.

Perino L.J. (1997): A guide to colostrum management in beef cows and calves. Vet. Med., 85-82.

Ragbetli T.P., Yoruk I. and Cay M. (2009): The effect of tulathoromycin treatment on antioxidant vitamins in montofen calves with pneumonia. Journal and Veterinary Advances, 8 (11): 2345-2349.

Richeson J.T., Pinedo P.J., Kegley E.B., Powell J.G., Gadberry M.S., Beck P.A. and Falkenberg S.M. (2013): Association of hematologic variables and castration status at the time of arrival at a research facility with the risk of bovine respiratory disease in beef calves. JAVMA., 243 (7): 1035-1041.

Skogerboe T.L., Rooney K.A., Weigel D.J. and Kilgore W.R. (2005): Comparative efficacy of tulathromycin versus florfenicol and tilmicosin against undifferentiated bovine respiratory disease in feedlot cattle. Vet. There, 6 (2): 180-196.

Thiry J., González-Martín J.V., Elvira L., Pagot E., Voisin F., Lequeux G., Weingarten A. and Haas V.D. (2014): Treatment of naturally occurring bovine respiratory disease in juvenile calves with a single administration of a florfenicol plus flunixin meglumine formulation. Vet. Rec., 174-430. 